\title{
LAI and Leaf Biomass Allometric Equations for Three Common Tree Species in a Hyrcanian Temperate Forest
}

\author{
Saleh Kahyani ${ }^{1}$, Hormoz Sohrabi ${ }^{*}$, Seyed Mohsen Hosseini' ${ }^{1}$, Jerry Vanclay ${ }^{2}$ \\ ${ }^{1}$ Department of Forestry, Faculty of Natural Resources and Marine Sciences, Tarbiat Modares University, \\ Noor, Mazandaran, Iran \\ ${ }^{2}$ Forest Research Centre, School of Environment, Science and Engineering, Southern Cross University, \\ Lismore, NSW 2480, Australia \\ Email: skahyani@yahoo.com, *hsohrabi@modares.ac.ir, hosseini@modares.ac.ir, Jerry.Vanclay@scu.edu.au
}

Received 1 March 2016; accepted 4 April 2016; published 7 April 2016

Copyright (C) 2016 by authors and Scientific Research Publishing Inc.

This work is licensed under the Creative Commons Attribution International License (CC BY). http://creativecommons.org/licenses/by/4.0/

(c) (i) Open Access

\section{Abstract}

Despite the importance of Hyrcanian forests for biodiversity conservation, a few studies with biomass destruction has been done to predict biomass and carbon pools from this forest and there is a lack of knowledge in our country. Biomass and leaf area index (LAI) are important variables in many ecological and environmental applications and forest management. In this paper, allometric biomass and leaf area equations were developed for three common Hyrcanian tree species, Oriental Beech (Fagus orientalis Lipsky), Hornbeam (Carpinus Betulus Lipsky) and Chestnutleaved Oak (Quercus castaneifolia C. A. Mey). To evaluate and estimate the leaf biomass and leaf area index of Oriental Beech, Horbeam and Chestnut-leaved Oak, 21, 27 and 17 individuals were selected and felled down, respectively. Tree characteristics such as diameter at breast height, total height, crown length and perpendicular diameters were measured. Destructive sampling was applied for determination of leaf biomass and LAI. Allometric equations were calculated for estimation of leaf biomass and LAI using simple linear regression and nonlinear regression analysis. The equations were compared based on several modelling parameters. Model comparison and selection were based on $\mathrm{R}^{2}$, Akaike's information criterion (AIC), prediction error sums of squares, model standard error estimate (SEE), $\triangle \mathrm{AIC}$, and a correction factor. Based on the results, the mean values of leaf area, leaf biomass and LAI for Oriental Beech were $53.05 \mathrm{~cm}^{2}, 0.176 \mathrm{gr}, 2.16$, for Hornbeam were $27.2 \mathrm{~cm}^{2}, 0.128 \mathrm{gr}, 1.13$ and for Chestnut-leaved Oak were $62.419 \mathrm{~cm}^{2}, 0.401 \mathrm{gr}$, 2.26, respectively. The highest significant correlation for Oriental Beech was found between LAI and total height $\left(\mathrm{R}^{2}\right.$ adj $=\mathbf{0}$. 931), the highest significant correlation for Hornbeam was found between LAI and Dbh $\left(R^{2}\right.$ adj $\left.=0.956\right)$ and the highest significance for Chestnut-leaved Oak was found between LAI and SqrtDbh $\left(R^{2}{ }^{a d j}=0.956\right)$. Also, the best equations were obtained by means of an

\footnotetext{
${ }^{*}$ Corresponding author.
}

How to cite this paper: Kahyani, S., Sohrabi, H., Hosseini, S. M., \& Vanclay, J. (2016). LAI and Leaf Biomass Allometric Equations for Three Common Tree Species in a Hyrcanian Temperate Forest. Open Journal of Forestry, 6, 1-7. 
exponential regression model for Oriental Beech, the Log-transformed regression model for Hornbeam and of a transformed regression model for Chestnut-leaved Oak.

\title{
Keywords
}

\author{
Leaf Biomass, LAI, Hyrcanian Forests, Fagus Orientalis, Carpinus Betulus, Quercus Castaneifolia
}

\section{Introduction}

Physiologists and ecologists have long recognized the importance of both leaf surface area and foliar weight as factors affecting many trees and stand-level processes and functions such as photosynthesis, gas exchange, growth, stand productivity and canopy dynamics (Johansson, 2002; Karlik and McKay, 2002; Eriksson et al., 2005; Pourhashemi et al., 2012). The leaf area is the main surface of the exchange of energy and matter, between the plant canopy and the atmosphere, and therefore a key variable in driving the biological processes of the plants (Eriksson et al., 2005; Pourhashemi et al., 2012). By measuring the leaf area, it is possible to estimate parameters like canopy structure (Welles and Norman, 1991) and canopy productivity (Dantec et al., 2000). The leaf area index, or LAI, defined as half the total leaf area per unit ground surface area (Fournier et al., 2003; Menzies et al., 2006; kigomo et al., 2013) is an important canopy variable needed for many physiological and ecosystem studies (Menzies et al., 2006), is also a useful "bulk" parameter which is a necessary input parameter in many models. It has been related to canopy interception, transpiration, net photosynthesis and net primary productivity (Botkin, 1986; Pierce \& Running, 1988; Gholz et al., 1991; Gower \& Norman, 1991; Kigomo et al., 2013). LAI is related to atmospheric gas exchange that controls local carbon cycling. Leaf retention of gases such as $\mathrm{CO}_{2}$ in the canopy is directly related to the amount of carbon released into the atmosphere (White et al., 1997; Menzies et al., 2006). LAI is also a key variable in analyzing energy absorption for photosynthesis (Cournac et al., 2002; Menzies et al., 2006), rates of photosynthesis, and for estimating primary and net primary production (Jensen, 2000; Menzies et al., 2006). Hyrcanian forests which are located in the north of Iran stretch up to an altitude of $2800 \mathrm{~m}$ above sea level. They are constituted of different forest stands with about 80 woody species. The communities of oriental beech (Fagus orientalis Lipsky) forests are the most important parts of the Hyrcanian forests due to their valuable ecological characteristics and their commercial value. Traditionally, measuring LAI was highly destructive because trees had to be felled for accurate measurements (Menzies et al., 2006).The aim of this work is to evaluate and estimate the leaf biomass and LAI of Oriental Beech and Chestnut-leaved Oak. So allometric leaf biomass and leaf area index equations were developed for Oriental Beech (Fagus orientalis Lipsky), Hornbeam (Carpinus Betulus Lipsky) and Chestnut-leaved Oak (Quercu scastaneifolia C. A. Mey).

\section{Material and Methods}

\subsection{Study Area}

The study was carried out in Chamestan Forest Districts $\left(36^{\circ} 22^{\prime} 00^{\prime \prime}-36^{\circ} 27^{\prime} 35^{\prime \prime} \mathrm{N}\right.$ and $\left.52^{\circ} 02^{\prime} 30^{\prime \prime}-52^{\circ} 07^{\prime} 30^{\prime \prime E}\right)$ located in northern forests of Iran (Figure 1). The study land has a total area of 2762 ha with altitudes which range between 250 and $1000 \mathrm{~m}$ a.s.l. The studied forests belong to the Beech community widely distributed in most parts of the district. The abundant tree species in the studied forests are F. orientalis L., C. betulus L. and Q. scastaneifolia C.A.M. According to data of the Nowshahr Meteorological Station, the mean annual precipitation and temperature of this area were $866 \mathrm{~mm}$ and $16.1^{\circ} \mathrm{C}$, respectively.

\subsection{Sampling and Data Analysis}

A total of 65 sample trees belonging to F. orientalis L. (21 individuals), C. betulus L. (27 individuals) and Quercu scastaneifolia C. A. Mey (17 individuals) were selected and felled down for leaf biomass and LAI estimation and allometric equation parameterization in the study area. All the trees were selected following lines of exploitation carried out by the forestry department of Nowshahr. The selection of each individual tree was based on diameter at breast height (1.3 m above the ground). The individuals were grouped into three DBH classes: 30 
- 60, 60 - 80 and $\geq 80 \mathrm{~cm}$, which is the diameter classification system commonly used in the Hyrcanian forests of Iran (Vahedi et al., 2013). Tree characteristics such as diameter at breast height, total height, crown length and perpendicular diameters were measured. Destructive sampling was applied for determination of leaf biomass and LAI. Gravimetric method was applied for calculating LAI (Adl, 2007). Allometric equations were calculated for estimation of leaf biomass and LAI using simple linear regression and nonlinear regression analysis. The equations were compared based on several modelling parameters. Model comparison and selection were based on $\mathrm{R}^{2}$, Akaike's Information Criterion (AIC), prediction error sums of squares, model standard error estimate (SEE), $\triangle \mathrm{AIC}$, and correction factor (Sohrabi \& Shirvani, 2012; Vahedi et al., 2013).

\section{Results and Discussion}

The mean values of DBH, height and crown area as measurable characteristics related to the subset of trees that was destructively sampled for leaf biomass and LAI estimation are summarized in Table 1. The diameter (DBH) range of the felled trees spanned from 17.19 to $108.5 \mathrm{~cm}$ so as to represent the diameter distribution reported in the studied stands. Based on the results, the mean values of leaf area, leaf biomass and LAI for Oriental Beech were $53.05 \mathrm{~cm}^{2}, 0.176 \mathrm{gr}, 2.16$, for Hornbeam were $27.2 \mathrm{~cm}^{2}, 0.128 \mathrm{gr}, 1.13$ and for Chestnut-leaved Oak were $62.419 \mathrm{~cm}^{2}, 0.401 \mathrm{gr}, 2.26$, respectively (Table 1).

Regression between the Leaf biomass (gr) and the Leaf Area $\left(\mathrm{cm}^{2}\right)$ of sampled trees are shown in Figure 2. A simple linear regression model using leaf biomass as independent variable showed significant correlation and produced $\mathrm{R}^{2}$ of $0.931,0.969$ and 0.981 for Oriental Beech, Hornbeam and Chestnut-leaved Oak, respectively (Figure 2).

Univariate linear and non-linear regression models were developed using measured variables. Linear and non-linear regression analysis were parameterized to predict the values of LAI using a set of allometric parameters including DBH, height, crown area and their combinations of sampling trees of Oriental Beech, Hornbeam and Chestnut-leaved Oak (Table 2 and Table 3).
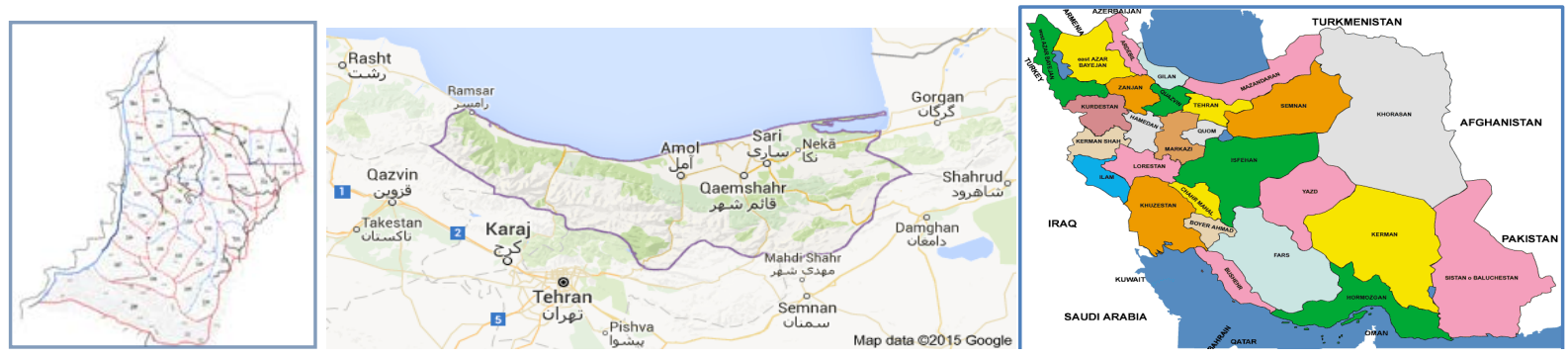

Figure 1. Location of the study area.

Table 1. Sample trees and leaf characteristics of the oriental beech, hornbeam and chestnut-leaved Oak.

\begin{tabular}{|c|c|c|c|c|c|c|c|}
\hline Species & & Dbh (cm) & $\mathrm{H}(\mathrm{m})$ & Leaf Biomass (gr) & Mean Leaf Area $\left(\mathrm{cm}^{2}\right)$ & $\mathrm{CA}\left(\mathrm{m}^{2}\right)$ & LAI \\
\hline \multirow{3}{*}{ Oriental Beech } & Minimum & 22.63 & 19.10 & 0.044 & 44.15 & 37.82 & 0.64 \\
\hline & Maximum & 108.50 & 34.85 & 0.355 & 66.66 & 120 & 3.66 \\
\hline & Mean & 66.56 & 28.94 & 0.176 & 53.05 & 84.43 & 2.16 \\
\hline \multirow{3}{*}{ Hornbeam } & Minimum & 22.14 & 13.60 & 0.028 & 14.36 & 24.90 & 0.25 \\
\hline & Maximum & 106.50 & 29.05 & 0.289 & 37.64 & 119.54 & 2.78 \\
\hline & Mean & 58.59 & 22.57 & 0.128 & 27.20 & 82.46 & 1.13 \\
\hline \multirow{3}{*}{$\begin{array}{c}\text { Chestnut-leaved } \\
\text { Oak }\end{array}$} & Minimum & 17.19 & 16.27 & 0.139 & 42.20 & 25.86 & 0.58 \\
\hline & Maximum & 72.85 & 29.83 & 0.867 & 88.05 & 99.30 & 4.27 \\
\hline & Mean & 42.49 & 24.60 & 0.401 & 62.419 & 58.57 & 2.26 \\
\hline
\end{tabular}


Table 2. Linear Regression and model description based on Dbh, height and crown area for the estimation of LAI of oriental beech, hornbeam and chestnut-leaved oak.

\begin{tabular}{|c|c|c|c|c|c|c|c|c|c|c|}
\hline Species & Allometric model & $\mathrm{R}$ & $\mathrm{R}_{\text {(adj) }}^{2}$ & a & $\mathrm{b}$ & DW & CF & $\begin{array}{l}\text { Std } \\
\text { Error }\end{array}$ & RMSE & Sig \\
\hline \multirow{15}{*}{ Oriental Beech } & $\mathrm{LAI}=\mathrm{a}(\mathrm{Dbh})+\mathrm{b}$ & 0.957 & 0.912 & 0.041 & -0.548 & 1.190 & - & 0.30249 & 0.5500 & ${ }^{* * *}$ \\
\hline & $\mathrm{LAI}=\mathrm{a}(\mathrm{h})+\mathrm{b}$ & 0.951 & 0.900 & 0.203 & -3.726 & 1.403 & - & 0.32272 & 0.5681 & *** \\
\hline & $\mathrm{LAI}=\mathrm{a}(\mathrm{CA})+\mathrm{b}$ & 0.898 & 0.798 & 0.046 & -1.692 & 1.039 & - & 0.45901 & 0.6775 & ${ }^{* * *}$ \\
\hline & $\mathrm{LAI}=\mathrm{a}(\mathrm{Dbh} \times \mathrm{h})+\mathrm{b}$ & 0.958 & 0.914 & 0.001 & 0.136 & 1.247 & - & 0.29902 & 0.5468 & ${ }^{* * *}$ \\
\hline & $\mathrm{LAI}=\mathrm{a}\left(\mathrm{Dbh} \times \mathrm{h}^{2}\right)+\mathrm{b}$ & 0.952 & 0.903 & $2.95 \times 10^{-5}$ & 0.52 & 1.345 & - & 0.31897 & 0.5648 & *** \\
\hline & $\mathrm{LAI}=\mathrm{a}\left((\mathrm{Dbh} \times \mathrm{h})^{2}\right)+\mathrm{b}$ & 0.909 & 0.820 & $2.19 \times 10^{-7}$ & 1.051 & 1.533 & - & 0.43337 & 0.6583 & ${ }^{* * *}$ \\
\hline & $\mathrm{LAI}=\mathrm{a}(\sqrt{\mathrm{Dbh}})+\mathrm{b}$ & 0.951 & 0.901 & 0.628 & -2.871 & 1.258 & - & 0.32182 & 0.5673 & *** \\
\hline & $\mathrm{LAI}=\mathrm{a}(\sqrt{\mathrm{h}})+\mathrm{b}$ & 0.942 & 0.883 & 2.101 & -9.098 & 1.464 & - & 0.34903 & 0.5908 & *** \\
\hline & $\mathrm{LAI}=\mathrm{a}(\sqrt{\mathrm{Dbh} \times \mathrm{h}})+\mathrm{b}$ & 0.959 & 0.916 & 0.085 & -1.527 & 1.237 & - & 0.29711 & 0.5451 & *** \\
\hline & $\mathrm{LAI}=\mathrm{a}\left(\sqrt{\mathrm{Dbh} \times \mathrm{h}^{2}}\right)+\mathrm{b}$ & 0.962 & 0.923 & 0.012 & -0.787 & 1.213 & - & 0.28375 & 0.5327 & *** \\
\hline & $\operatorname{Ln}(\mathrm{LAI})=\mathrm{a} \operatorname{Ln}(\mathrm{Dbh})+\mathrm{b}$ & 0.955 & 0.909 & 1.320 & -4.810 & 1.097 & 1.0152 & 0.17384 & 0.4169 & ${ }^{* * *}$ \\
\hline & $\operatorname{Ln}(\mathrm{LAI})=\mathrm{a} \operatorname{Ln}(\mathrm{h})+\mathrm{b}$ & 0.963 & 0.924 & 3.131 & -9.861 & 1.173 & 1.0128 & 0.15957 & 0.3995 & ${ }^{* * *}$ \\
\hline & $\operatorname{Ln}(\mathrm{LAI})=\mathrm{a} \operatorname{Ln}(\mathrm{Dbh} \times \mathrm{h})+\mathrm{b}$ & 0.959 & 0.917 & 0.931 & -6.331 & 1.120 & 1.0140 & 0.16661 & 0.4082 & *** \\
\hline & $\operatorname{Ln}(\mathrm{LAI})=\mathrm{a} \operatorname{Ln}\left(\mathrm{Dbh} \times \mathrm{h}^{2}\right)+\mathrm{b}$ & 0.961 & 0.920 & 0.719 & -7.151 & 1.133 & 1.0135 & 0.16368 & 0.4046 & $* * *$ \\
\hline & $\operatorname{Ln}(\mathrm{LAI})=\mathrm{a} \operatorname{Ln}\left((\mathrm{Dbh} \times \mathrm{h})^{2}\right)+\mathrm{b}$ & 0.959 & 0.917 & 0.466 & -6.331 & 1.120 & 1.0140 & 0.16661 & 0.4082 & *** \\
\hline \multirow{15}{*}{ Hornbeam } & $\mathrm{LAI}=\mathrm{a}(\mathrm{Dbh})+\mathrm{b}$ & 0.910 & 0.823 & 0.027 & -0.457 & 2.152 & - & 0.31266 & 0.5592 & *** \\
\hline & $\mathrm{LAI}=\mathrm{a}(\mathrm{h})+\mathrm{b}$ & 0.846 & 0.706 & 0.148 & -2.217 & 1.974 & - & 0.40277 & 0.6346 & *** \\
\hline & $\mathrm{LAI}=\mathrm{a}(\mathrm{CA})+\mathrm{b}$ & 0.884 & 0.773 & 0.014 & -0.020 & 2.468 & - & 0.35408 & 0.5950 & $* * *$ \\
\hline & $\mathrm{LAI}=\mathrm{a}(\mathrm{Dbh} \times \mathrm{h})+\mathrm{b}$ & 0.915 & 0.832 & 0.001 & -0.041 & 2.223 & - & 0.30496 & 0.5522 & ${ }^{* * *}$ \\
\hline & $\mathrm{LAI}=\mathrm{a}\left(\mathrm{Dbh} \times \mathrm{h}^{2}\right)+\mathrm{b}$ & 0.919 & 0.840 & $2.69 \times 10^{-5}$ & 0.179 & 2.323 & - & 0.29715 & 0.5451 & ${ }^{* * *}$ \\
\hline & $\mathrm{LAI}=\mathrm{a}\left((\mathrm{Dbh} \times \mathrm{h})^{2}\right)+\mathrm{b}$ & 0.923 & 0.847 & $2.48 \times 10^{-7}$ & 0.465 & 2.594 & - & 0.29031 & 0.5388 & *** \\
\hline & $\mathrm{LAI}=\mathrm{a}(\sqrt{\mathrm{Dbh}})+\mathrm{b}$ & 0.976 & 0.956 & 0.405 & -1.899 & 2.060 & - & 0.15258 & 0.3853 & *** \\
\hline & $\mathrm{LAI}=\mathrm{a}(\sqrt{\mathrm{h}})+\mathrm{b}$ & 0.831 & 0.679 & 1.353 & -5.269 & 1.944 & - & 0.42082 & 0.6487 & ${ }^{* * *}$ \\
\hline & $\mathrm{LAI}=\mathrm{a}(\sqrt{\mathrm{Dbh} \times \mathrm{h}})+\mathrm{b}$ & 0.891 & 0.788 & 0.060 & -1.030 & 2.066 & - & 0.34257 & 0.5853 & *** \\
\hline & $\mathrm{LAI}=\mathrm{a}\left(\sqrt{\mathrm{Dbh} \times \mathrm{h}^{2}}\right)+\mathrm{b}$ & 0.895 & 0.795 & 0.010 & -0.576 & 2.092 & - & 0.33687 & 0.5804 & ${ }^{* * *}$ \\
\hline & $\operatorname{Ln}(\mathrm{LAI})=\mathrm{a} \operatorname{Ln}(\mathrm{Dbh})+\mathrm{b}$ & 0.978 & 0.956 & 1.388 & -5.605 & 2.110 & 1.0318 & 0.15009 & 0.3801 & *** \\
\hline & $\operatorname{Ln}(\mathrm{LAI})=\mathrm{a} \operatorname{Ln}(\mathrm{h})+\mathrm{b}$ & 0.909 & 0.820 & 3.054 & -9.548 & 1.806 & 1.0408 & 0.28278 & 0.5318 & ${ }^{* * *}$ \\
\hline & $\operatorname{Ln}(\mathrm{LAI})=\mathrm{a} \operatorname{Ln}(\mathrm{Dbh} \times \mathrm{h})+\mathrm{b}$ & 0.925 & 0.851 & 0.959 & -6.872 & 1.995 & 1.0335 & 0.25686 & 0.5068 & *** \\
\hline & $\operatorname{Ln}(\mathrm{LAI})=\mathrm{a} \operatorname{Ln}\left(\mathrm{Dbh} \times \mathrm{h}^{2}\right)+\mathrm{b}$ & 0.922 & 0.846 & 0.732 & -7.527 & 1.942 & 1.0348 & 0.26173 & 0.5116 & ${ }^{* * *}$ \\
\hline & $\operatorname{Ln}(\mathrm{LAI})=\mathrm{a} \operatorname{Ln}\left((\mathrm{Dbh} \times \mathrm{h})^{2}\right)+\mathrm{b}$ & 0.925 & 0.851 & 0.480 & -6.872 & 1.995 & 1.0335 & 0.25686 & 0.5068 & ${ }^{* * *}$ \\
\hline \multirow{4}{*}{$\begin{array}{c}\text { Chestnut-leaved } \\
\text { Oak }\end{array}$} & $\mathrm{LAI}=\mathrm{a}(\mathrm{Dbh})+\mathrm{b}$ & 0.975 & 0.948 & 0.058 & -0.221 & 1.836 & - & 0.23267 & 0.4824 & *** \\
\hline & $\mathrm{LAI}=\mathrm{a}(\mathrm{h})+\mathrm{b}$ & 0.964 & 0.924 & 0.248 & -3.837 & 2.843 & - & 0.28142 & 0.5305 & $* * *$ \\
\hline & $\mathrm{LAI}=\mathrm{a}(\mathrm{CA})+\mathrm{b}$ & 0.966 & 0.929 & 0.041 & -0.135 & 2.172 & - & 0.27201 & 0.5215 & *** \\
\hline & $\mathrm{LAI}=\mathrm{a}(\mathrm{Dbh} \times \mathrm{h})+\mathrm{b}$ & 0.972 & 0.941 & 0.002 & 0.399 & 1.711 & - & 0.24708 & 0.4971 & *** \\
\hline
\end{tabular}




\section{Continued}

\begin{tabular}{|c|c|c|c|c|c|c|c|c|c|}
\hline $\mathrm{LAI}=\mathrm{a}\left(\mathrm{Dbh} \times \mathrm{h}^{2}\right)+\mathrm{b}$ & 0.966 & 0.930 & $5.18 \times 10^{-5}$ & 0.746 & 1.579 & - & 0.27043 & 0.5200 & $* * *$ \\
\hline $\mathrm{LAI}=\mathrm{a}\left((\mathrm{Dbh} \times \mathrm{h})^{2}\right)+\mathrm{b}$ & 0.937 & 0.870 & $6.49 \times 10^{-7}$ & 1.260 & 1.486 & - & 0.36784 & 0.6065 & ${ }^{* * *}$ \\
\hline $\mathrm{LAI}=\mathrm{a}(\sqrt{\mathrm{Dbh}})+\mathrm{b}$ & 0.976 & 0.956 & 0.762 & -2.609 & 2.403 & - & 0.21355 & 0.4621 & ${ }^{* * *}$ \\
\hline $\mathrm{LAI}=\mathrm{a}(\sqrt{\mathrm{h}})+\mathrm{b}$ & 0.957 & 0.910 & 2.382 & -9.510 & 2.787 & - & 0.30636 & 0.5535 & ${ }^{* * *}$ \\
\hline $\mathrm{LAI}=\mathrm{a}(\sqrt{\mathrm{Dbh} \times \mathrm{h}})+\mathrm{b}$ & 0.979 & 0.955 & 0.111 & -1.306 & 2.430 & - & 0.21533 & 0.4640 & ${ }^{* * *}$ \\
\hline $\mathrm{LAI}=\mathrm{a}\left(\sqrt{\mathrm{Dbh} \times \mathrm{h}^{2}}\right)+\mathrm{b}$ & 0.978 & 0.954 & 0.018 & -0.582 & 2.339 & - & 0.21863 & 0.4676 & ${ }^{* * *}$ \\
\hline $\operatorname{Ln}(\mathrm{LAI})=\mathrm{a} \operatorname{Ln}(\mathrm{Dbh})+\mathrm{b}$ & 0.896 & 0.790 & 0.029 & -0.557 & 1.586 & 1.0333 & 0.25604 & 0.5060 & ${ }^{* * *}$ \\
\hline $\operatorname{Ln}(\mathrm{LAI})=\mathrm{a} \operatorname{Ln}(\mathrm{h})+\mathrm{b}$ & 0.962 & 0.921 & 0.136 & -2.644 & 2.163 & 1.0124 & 0.15725 & 0.3965 & ${ }^{* * *}$ \\
\hline $\operatorname{Ln}(\mathrm{LAI})=\mathrm{a} \operatorname{Ln}(\mathrm{Dbh} \times \mathrm{h})+\mathrm{b}$ & 0.886 & 0.770 & 0.001 & -0.237 & 1.557 & 1.0365 & 0.26782 & 0.5175 & ${ }^{* * *}$ \\
\hline $\operatorname{Ln}(\mathrm{LAI})=\mathrm{a} \operatorname{Ln}\left(\mathrm{Dbh} \times \mathrm{h}^{2}\right)+\mathrm{b}$ & 0.869 & 0.739 & $2.55 \times 10^{-5}$ & -0.054 & 1.531 & 1.0414 & 0.28499 & 0.5338 & *** \\
\hline $\operatorname{Ln}(\mathrm{LAI})=\mathrm{a} \operatorname{Ln}\left((\mathrm{Dbh} \times \mathrm{h})^{2}\right)+\mathrm{b}$ & 0.802 & 0.619 & $3.04 \times 10^{-7}$ & 0.224 & 1.539 & 1.0612 & 0.34463 & 0.5871 & *** \\
\hline
\end{tabular}

Table 3. Nonlinear regression and Model description based on DBH, height and crown area for the estimation of LAI of oriental beech, hornbeam and Chestnut-leaved Oak.

\begin{tabular}{|c|c|c|c|c|c|c|c|c|c|c|c|}
\hline Species & $\mathrm{X}_{\mathrm{var}}$ & Allometric Model & $\mathrm{R}$ & $\mathrm{R}_{(\text {(adj) }}^{2}$ & a & $\mathrm{b}$ & $\mathrm{CF}$ & $\begin{array}{l}\text { Std. } \\
\text { Error }\end{array}$ & RMSE & F & Sig \\
\hline \multirow{9}{*}{ Oriental Beech } & \multirow{3}{*}{ Dbh } & $\mathrm{LAI}=\mathrm{a} \log (\mathrm{Dbh})+\mathrm{b}$ & 0.930 & 0.859 & 2.274 & -7.216 & 1.0765 & 0.384 & 0.6197 & 158.861 & ${ }^{* * *}$ \\
\hline & & $\mathrm{LAI}=\mathrm{a}(\mathrm{Dbh})^{\mathrm{b}}$ & 0.955 & 0.909 & 1.320 & 0.008 & 1.0153 & 1.174 & 0.4171 & 261.509 & ${ }^{* * *}$ \\
\hline & & $\mathrm{LAI}=\operatorname{aexp}[\mathrm{a}(\mathrm{Dbh})]$ & 0.942 & 0.883 & 0.023 & 0.417 & 1.0198 & 0.198 & 0.4450 & 196.767 & ${ }^{* * *}$ \\
\hline & \multirow{3}{*}{ h } & $\mathrm{LAI}=\mathrm{a} \log (\mathrm{h})+\mathrm{b}$ & 0.931 & 0.862 & 5.367 & -15.822 & 1.0745 & 0.379 & 0.6156 & 163.790 & ${ }^{* * *}$ \\
\hline & & $\mathrm{LAI}=\mathrm{a}(\mathrm{h})^{\mathrm{b}}$ & 0.963 & 0.924 & 3.131 & $5.22 \times 10^{-5}$ & 1.0129 & 0.160 & 0.4000 & 315.045 & $* * *$ \\
\hline & & $\mathrm{LAI}=\mathrm{a} \exp [\mathrm{a}(\mathrm{h})]$ & 0.966 & 0.931 & 0.117 & 0.064 & 1.0115 & 0.151 & 0.3886 & 352.636 & $* * *$ \\
\hline & \multirow{3}{*}{ CA } & $\mathrm{LAI}=\mathrm{a} \log (\mathrm{CA})+\mathrm{b}$ & 0.848 & 0.709 & 3.160 & -11.754 & 1.1646 & 0.522 & 0.7430 & 64.211 & ${ }^{* * *}$ \\
\hline & & $\mathrm{LAI}=\mathrm{a}(\mathrm{CA})^{\mathrm{b}}$ & 0.878 & 0.762 & 1.846 & 0.001 & 1.0406 & 0.282 & 0.5310 & 84.068 & ${ }^{* * *}$ \\
\hline & & $\mathrm{LAI}=\operatorname{aexp}[\mathrm{a}(\mathrm{CA})]$ & 0.895 & 0.792 & 0.026 & 0.215 & 1.0352 & 0.263 & 0.5128 & 100.171 & ${ }^{* * * *}$ \\
\hline \multirow{9}{*}{ Hornbeam } & \multirow{3}{*}{ Dbh } & $\mathrm{LAI}=\mathrm{a} \log (\mathrm{Dbh})+\mathrm{b}$ & 0.864 & 0.738 & 1.440 & -4.597 & 1.0749 & 0.380 & 0.6164 & 85.473 & ${ }^{* * * *}$ \\
\hline & & $\mathrm{LAI}=\mathrm{a}(\mathrm{Dbh})^{\mathrm{b}}$ & 0.929 & 0.859 & 1.388 & 0.004 & 1.0317 & 0.250 & 0.5000 & 183.917 & ${ }^{* * *}$ \\
\hline & & $\mathrm{LAI}=\operatorname{aexp}[\mathrm{a}(\mathrm{Dbh})]$ & 0.918 & 0.838 & 0.024 & 0.219 & 1.0366 & 0.268 & 0.5177 & 156.202 & ${ }^{* * *}$ \\
\hline & \multirow{3}{*}{$\mathrm{h}$} & $\mathrm{LAI}=\mathrm{alog}(\mathrm{h})+\mathrm{b}$ & 0.813 & 0.650 & 3.049 & -8.316 & 1.1016 & 0.440 & 0.6633 & 56.598 & ${ }^{* * *}$ \\
\hline & & $\mathrm{LAI}=\mathrm{a}(\mathrm{h})^{\mathrm{b}}$ & 0.909 & 0.820 & 3.054 & $7.14 \times 10^{-5}$ & 1.0409 & 0.283 & 0.5320 & 137.539 & ${ }^{* * * *}$ \\
\hline & & $\mathrm{LAI}=\operatorname{aexp}[\mathrm{a}(\mathrm{h})]$ & 0.919 & 0.839 & 0.144 & 0.035 & 1.0363 & 0.267 & 0.5167 & 157.306 & ${ }^{* * *}$ \\
\hline & \multirow{3}{*}{ CA } & $\mathrm{LAI}=\mathrm{a} \log (\mathrm{CA})+\mathrm{b}$ & 0.836 & 0.668 & 1.020 & -3.196 & 1.0899 & 0.415 & 0.6442 & 67.107 & ${ }^{* * *}$ \\
\hline & & $\mathrm{LAI}=\mathrm{a}(\mathrm{CA})^{\mathrm{b}}$ & 0.893 & 0.790 & 0.015 & 0.977 & 1.0476 & 0.305 & 0.5523 & 113.763 & ${ }^{* * *}$ \\
\hline & & $\mathrm{LAI}=\operatorname{aexp}[\mathrm{a}(\mathrm{CA})]$ & 0.876 & 0.760 & 0.012 & 0.331 & 1.0546 & 0.326 & 0.5710 & 96.039 & ${ }^{* * *}$ \\
\hline \multirow{9}{*}{$\begin{array}{c}\text { Chestnut-leaved } \\
\text { Oak }\end{array}$} & \multirow{3}{*}{ Dbh } & $\mathrm{LAI}=\mathrm{a} \log (\mathrm{Dbh})+\mathrm{b}$ & 0.975 & 0.947 & 2.377 & -6.460 & 1.0278 & 0.234 & 0.4837 & 288.591 & ${ }^{* * * *}$ \\
\hline & & $\mathrm{LAI}=\mathrm{a}(\mathrm{Dbh})^{\mathrm{b}}$ & 0.950 & 0.896 & 1.269 & 0.019 & 1.0163 & 0.180 & 0.4243 & 139.439 & $* * *$ \\
\hline & & $\mathrm{LAI}=\operatorname{aexp}[\mathrm{a}(\mathrm{Dbh})]$ & 0.896 & 0.790 & 0.029 & 0.573 & 1.0333 & 0.256 & 0.506 & 61.031 & ${ }^{* * *}$ \\
\hline & \multirow{3}{*}{$\mathrm{h}$} & $\mathrm{LAI}=\mathrm{alog}(\mathrm{h})+\mathrm{b}$ & 0.948 & 0.891 & 5.662 & -15.794 & 1.0581 & 0.336 & 0.5797 & 132.074 & ${ }^{* * * *}$ \\
\hline & & $\mathrm{LAI}=\mathrm{a}(\mathrm{h})^{\mathrm{b}}$ & 0.971 & 0.939 & 3.179 & $7.92 \times 10^{-5}$ & 1.0096 & 0.138 & 0.3715 & 247.238 & ${ }^{* * *}$ \\
\hline & & $\mathrm{LAI}=\operatorname{aexp}[\mathrm{a}(\mathrm{h})]$ & 0.962 & 0.921 & 0.136 & 0.071 & 1.0124 & 0.157 & 0.3962 & 186.564 & ${ }^{* * *}$ \\
\hline & \multirow{3}{*}{ CA } & $\mathrm{LAI}=\mathrm{alog}(\mathrm{CA})+\mathrm{b}$ & 0.963 & 0.922 & 2.270 & -6.782 & 1.0412 & 0.284 & 0.5329 & 190.223 & ${ }^{* * *}$ \\
\hline & & $\mathrm{LAI}=\mathrm{a}(\mathrm{CA})^{\mathrm{b}}$ & 0.927 & 0.849 & 1.197 & 0.017 & 1.0238 & 0.217 & 0.4658 & 91.014 & $* * *$ \\
\hline & & $\mathrm{LAI}=\operatorname{aexp}[\mathrm{a}(\mathrm{CA})]$ & 0.886 & 0.771 & 0.021 & 0.600 & 1.0363 & 0.267 & 0.5167 & 54.773 & ${ }^{* * * *}$ \\
\hline
\end{tabular}




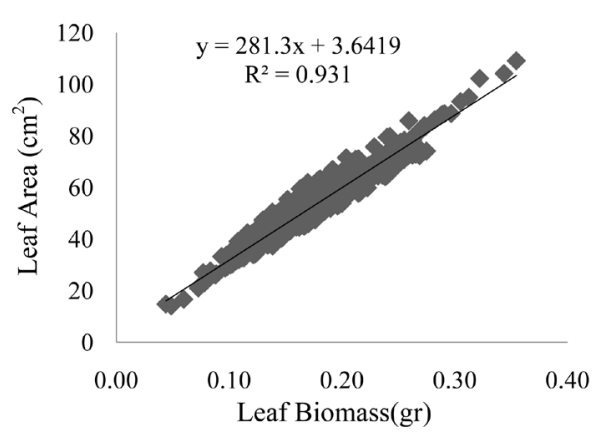

(a)

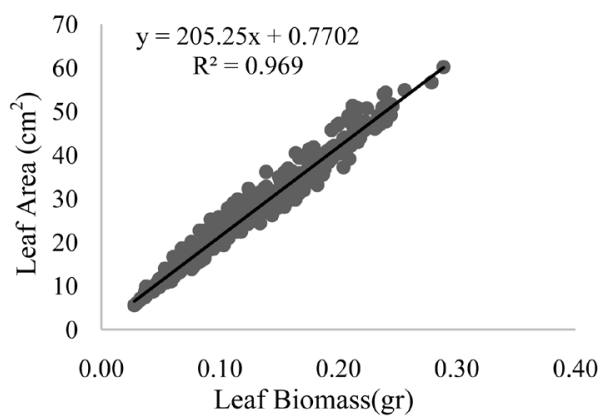

(b)

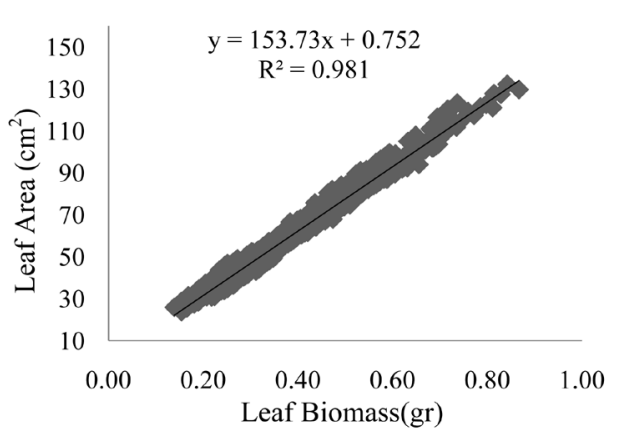

(c)

Figure 2. Regression between the leaf biomass (gr) and the Leaf Area $\left(\mathrm{cm}^{2}\right)$ of: (a) Oriental Beech; (b) Hornbeam and (c) Chestnut-leaved Oak.

The best-fit equations had adjusted $\mathrm{R}^{2}$ values between 0.709 to 0.931 and RMSE values between 0.7430 and 0.3886 for Oriental Beech, $\mathrm{R}^{2}$ values between 0.650 to 0.956 and RMSE values between 0.6633 and 0.3801 for Hornbeam and $\mathrm{R}^{2}$ values between 0.619 to 0.956 and RMSE values between 0.5871 and 0.4621 for Chestnut-leaved Oak (Table 1 and Table 2).

Allometric equations for estimating LAI from DBH and height and their combinations were derived. Based on results the highest significant correlation for Oriental Beech was found between LAI and total height $\left(\mathrm{R}_{\text {adj }}^{2}=0\right.$. 931), the highest significant correlation for Hornbeam was found between LAI and $D b h\left(R_{\text {adj }}^{2}=0.956\right)$ and the highest significance for Chestnut-leaved Oak was found between LAI and SqrtDbh $\left(\mathrm{R}_{\text {adj }}^{2}=0\right.$. 956). Also, the best equations were obtained by means of an exponential regression model for Oriental Beech, the Log-transformed regression model for Hornbeam and of a transformed regression model for Chestnut-leaved Oak.

\section{Conclusions}

In the presence of complex environmental gradients, allometric equations can provide additional information to improve the knowledge of LAI distribution.

Allometric equations presented in this study associated with oriental beech, Hornbeam and Chestnut-leaved oak, the three common species in the Hyrcanian forests, may bring additional information for the LAI patterns and distribution. The allometric equations provide improved methods for predicting LAI, leaf biomass and also carbon storage in Hyrcanian forests from standard forestry measurements, which means these equations may be applied to historical and new forest data.

\section{References}

Adl, H. R. (2007). Estimation of Leaf Biomass and Leaf Area Index of Two Major Species in Yasuj Forests. Iranian Journal of Forest and Poplar Research, 15, 417-426.

Botkin, D. B. (1986). Remote Sensing of the Biosphere (135 p). National Academy of Sciences, Report of the Committee on Planetary Biology, Washington, DC: National Research Council.

Cournac, L., Dubois, M., Chave, J., \& Riera, B. (2002). Fast Determination of Light Availability and Leaf Area Index in 
Tropical Forests. Journal of Tropical Ecology, 18, 295-302. http://dx.doi.org/10.1017/S0266467402002201

Dantec, V. L., Dufrêne, E., \& Saugier, B. (2000). Interannual and Spatial Variation in Maximum Leaf Area Index of Temperate Deciduous Stands. Forest Ecology and Management, 134, 71-81. http://dx.doi.org/10.1016/S0378-1127(99)00246-7

Eriksson, H., Eklundh, L., Hall, K., \& Lindroth, A. (2005). Estimating LAI in Deciduous Forest Stands. Agricultural and Forest Meteorology, 129, 27-37. http://dx.doi.org/10.1016/j.agrformet.2004.12.003

Fournier, R. A., Mailly, D., Walter, J. N., \& Soudan, K. (2003). Indirect Measurements of Forest Canopy Structure from in Situ Optical Sensors. In M. A. Wulber, \& S. E. Franklin (Eds.), Remote Sensing of Forest Environments: Concepts and Case Studies, Massachusetts: Kluwer Academic Publishers, 77-113. http://dx.doi.org/10.1007/978-1-4615-0306-4_4

Gholz, H. L., Vogel, S. A., Cropper, W. P., McKelvey, K., Ewel, K. C., Teskey, R. O., \& Curran, P. J. (1991). Dynamics of Canopy Structure and Light Interception in Pinus elliottii Stands, North Florida. Ecological Monographs, 61, 33-51. http://dx.doi.org/10.2307/1942998

Gower, S. T., \& Norman, J. M. (1991). Rapid Estimation of Leaf Area Index in Conifer and Broadleaf Plantations. Ecology, 72, 1896-1900. http://dx.doi.org/10.2307/1940988

Jensen, R. R. (2000), Measurement Comparison and Use of Remotely Derived Leaf Area Index Predictors (133 p). Ph.D. Dissertation, University of Florida.

Johansson, T., (2002). Increment and Biomass in 26- to 91-Year-Old European Aspen and Some Practical Implications. Journal of Biomass and Bioenergy, 23, 245-255. http://dx.doi.org/10.1016/S0961-9534(02)00056-9

Karlik, J. F., \& McKay, M. K. (2002). Leaf Area Index, Leaf Mass Density, and Allometric Relationships Derived from Harvest of Blue Oaks in a California Oak Savanna. In R. B. Standiford et al. (Eds.), Proceedings of the Fifth Symposium on Oak Woodlands: Oaks in California's Challenging Landscape (pp. 719-729). General Technical Report, PSW-GTR-184, Albany, CA: Pacific Southwest Research Station, Forest Service, U.S. Department of Agriculture.

Kigomo, J. N., Sweda, T., \& Tsuzuki, H. (2013). Improved Model for Estimation of Leaf Area Index Using Airborne Laser Profiling. Journal of Horticulture and Forestry, 5, 53-60.

Menzies, J., Jensen, R., Brondizio, E., Moran, E. F., \& Mausel, P. (2006). Accuracy of Neural Network and Regression Leaf Area Estimators for the Amazon Basin. GIScience \& Remote Sensing, 43, 1-11.

Pierce, L. L., \& Running, S. W. (1988). Rapid Estimation of Coniferous Forest Leaf Area Index Using Portable Integrating Radiometer. Ecology, 69, 1762-1767. http://dx.doi.org/10.2307/1941154

Pourhashemi, M., Eskandari, S., Dehghani, M., Najafi, T., Asadi, A., \& Panahi, P. (2012). Biomass and Leaf Area Index of Caucasian Hackberry (Celtis caucasica Willd.) in Taileh Urban Forest, Sanandaj, Iran. Iranian Journal of Forest and Poplar Research, 19, 609-620.

Sohrabi, H., \& Shirvani, A. (2012). Allometric Equations for Estimating Standing Biomass of Atlantic Pistache (Pistacia atlantica var. mutica) in Khojir National Park. Iranian Journal of Forest, 4, 55-64.

Vahedi, A., Mataji, A., Babayi-Kafaki, S., Eshaghi-Rad, J., \& Hodjati, S. M. (2013). Modeling the Bole Mass of Beech (Fagus Orientalis Lipsky) through Allometric Equations within Hyrcanian Forests. Iranian Journal of Forest, 5, 309-322.

Welles, J. M., \& Norman, J. M. (1991). Instrument for Indirect Measurement of Canopy Architecture. Agronomy Journal, 83, 818-825. http://dx.doi.org/10.2134/agronj1991.00021962008300050009x

White, J., Running, S., Nemani, R., Keane, R., \& Ryan, K. (1997). Measurement of Remote Sensing of LAI in Rocky Mountain Montane Ecosytems. Canadian Journal of Forest Research, 27, 1714-1727. http://dx.doi.org/10.1139/x97-142 